\title{
Flat-histogram Monte Carlo as an efficient tool to evaluate adsorption processes involving rigid and deformable molecules
}

\author{
Matthew Witman, ${ }^{\dagger, \ddagger}$ Nathan A. Mahynski, 9 and Berend Smit*,,$\ddagger$ \\ $\dagger$ Department of Chemical and Biomolecular Engineering, University of California, Berkeley \\ 94720, USA \\ $\ddagger$ Laboratory of Molecular Simulation, Institut des Sciences et Ingénierie Chimiques, Valais, \\ École Polytechnique Fédérale de Lausanne (EPFL), Rue de l'Industrie 17, CH-1951 Sion, \\ Switzerland \\ TChemical Sciences Division, National Institute of Standards and Technology, \\ Gaithersburg, Maryland 20899-8320, USA \\ E-mail: berend.smit@epfl.ch
}

\begin{abstract}
Monte Carlo simulations are the foundational technique for predicting thermodynamic properties of open systems where the process of interest involves the exchange of particles. Thus, they have been used extensively to computationally evaluate the adsorption properties of nanoporous materials and are critical for the in silico identification of promising materials for a variety of gas storage and chemical separation applications. In this work we demonstrate that a well-known biasing technique, known as "flat-histogram" sampling, can be combined with temperature extrapolation of the free energy landscape to efficiently provide significantly more useful thermodynamic information than standard open ensemble MC simulations. Namely, we can accurately
\end{abstract}


compute the isosteric heat of adsorption and number of particles adsorbed for various adsorbates over an extremely wide range of temperatures and pressures from a set of simulations at just one temperature. We extend this derivation of the temperature extrapolation to adsorbates with intramolecular degrees of freedom when Rosenbluth sampling is employed. Consequently, the working capacity and isosteric heat can be computed for any given combined temperature/pressure swing adsorption process for a large range of operating conditions with both rigid and deformable adsorbates. Continuous thermodynamic properties can be computed with this technique at very moderate computational cost, thereby providing a strong case for its application to the in silico identification of promising nanoporous adsorbents.

\section{Introduction}

Open ensemble Monte Carlo (MC) simulations, in which particles can be added or removed from a simulation domain, are the technique of choice for studying many important thermodynamic phenomena such as phase coexistence and adsorption. ${ }^{1}$ For example, Gibbs ensemble $\mathrm{MC}$ is particularly useful for predicting phase coexistence ${ }^{2,3}$ and grand canonical MC (GCMC) is often used to predict adsorption thermodynamics of fluids in porous media. ${ }^{4,5}$ Extensive work over the past several decades has focused on developing these methods, as well as biasing techniques to enhance sampling of systems where the standard acceptance probability of moves becomes prohibitively small. Some of the most prominent examples include Rosenbluth sampling, ${ }^{6}$ configurational bias MC (CBMC), ${ }^{7,8}$ and continuous fractional $\mathrm{MC}^{9}$ methods which allow effective sampling of deformable molecules and/or high density systems that otherwise cannot be simulated with standard GCMC particle insertion moves.

Another distinct category of biasing techniques emerged after Wang and Landau (WL) ${ }^{10}$ developed a general technique to construct a bias on-the-fly enabling the sampling of low probability (high free energy) states that are otherwise not sampled during a normal MC simulation. The goal of transition matrix Monte Carlo (TMMC) is closely related to that 
of the WL scheme, but there are distinct algorithmic differences between the two which are detailed extensively in the literature. ${ }^{11}$ These are generally referred to as flat-histogram or density-of-states sampling techniques since the goal is to sample states characterized by a given collective variable with uniform probability. ${ }^{12,13}$ Often the two techniques are merged to exploit the advantages of each method while avoiding their individual disadvantages, and have been applied extensively to study phase coexistence and adsorption thermodynamics. ${ }^{13-18}$ More complex applications of WL/TMMC have been developed recently, especially in the context of studying adsorption and phase coexistence in model systems that contain flexibility, multicomponent mixtures, and non-rigid molecules. ${ }^{19,20}$ Recently, an approach for extrapolating the free energy landscapes determined by WL/TMMC has presented an opportunity to predict continuous thermodynamic properties as a function of temperature by simply post-processing simulation data obtained at a single temperature. ${ }^{21-23}$

In this article, we build upon the aforementioned literature to demonstrate the effectiveness of a flat-histogram method for evaluating a porous material's performance in adsorption processes involving rigid and deformable adsorbates. By applying the recently developed temperature extrapolation procedure, ${ }^{21}$ we can predict the thermodynamic adsorption properties of a porous material over a continuous range of temperature and pressure conditions from a simulation at just one temperature. For the first time, we extend this derivation of temperature extrapolation to simulations in which Rosenbluth sampling has been used to sample deformable molecules (e.g., a flexible chain with internal degrees of freedom). This applicability to both rigid and flexible molecules, as well as the large temperature ranges over which thermodynamic properties can be extrapolated, demonstrates some significant advantages of this method compared to traditional GCMC for the in silico evaluation of porous materials. We additionally show how these techniques can be useful in practical applications. As an example, we consider the capture of gaseous species in nanoporous materials using combined temperature and pressure swing adsorption (T/PSA). If one would like to optimize the operating conditions for such an adsorption process, one needs to know 
the adsorption isotherms over a wide range of conditions. Using these flat-histogram and temperature extrapolation techniques, materials can be evaluated for T/PSA processes over a wide range of operating conditions (via determination of working capacities and isosteric heats) from simulation data obtained at just one temperature.

\section{Theoretical aspects}

A variety of studies have used flat-histogram approaches to determine the free energy landscape as a function of particle number in open ensembles. ${ }^{14,16,24,25}$ Due to the detailed discussion and derivation of these techniques elsewhere, we only briefly summarize the formalism to obtain the macrostate probabilities in the grand canonical ensemble. Then we show how analyzing the potential energy fluctuations at each macrostate allows determination of the free energy landscape at different temperatures by Taylor series expansion, and we subsequently extend this temperature extrapolation derivation to the case of deformable adsorbates. Finally we explain how to obtain the continuous thermodynamic properties of the adsorbate/adsorbent system that are important for predicting a material's utility as an adsorbent.

\section{Thermodynamic background}

We provide a brief review of the statistical mechanics equations that will be important for applying temperature extrapolation in the case when Rosenbluth sampling has been employed in conjunction with a TMMC biasing scheme. The total canonical partition function of an $N$ particle system in a given volume, $V$, at thermodynamic temperature, $\beta=\left(k_{B} T\right)^{-1}$ (where $k_{B}$ is Boltzmann's constant and $T$ is the absolute temperature), is denoted $Q(N, V, \beta)$. It can be factored into an integration over kinetic, $Q_{k}(N, \beta)$, and configurational, $Q_{c}(N, V, \beta)$, degrees of freedom as shown in Eqn. 1. 


$$
Q(N, V, \beta)=Q_{k}(N, \beta) Q_{c}(N, V, \beta)=Q_{k}(N, \beta)\left(\frac{1}{N !} \int d \mathbf{r}^{N} \exp \left[-\beta E\left(\Gamma_{\mathbf{r}^{N}}\right)\right]\right)
$$

Here $E$ is the total potential energy and $\Gamma_{\mathbf{r}^{N}}$ is the configuration of the $N$ particle system. More generally we can write $Q_{k}(N, \beta)=q(\beta)^{N}$, where $q(\beta)$ represents the integration over the kinetic degrees of freedom of a single molecule. In the case of monatomic particles, $q(\beta)=\Lambda^{-3}$ where $\Lambda$ is the thermal de Broglie wavelength; however, in what follows we keep the notation of $q(\beta)$ to more generally include polyatomic flexible molecules.

When this system can exchange particles with an infinite reservoir that imposes some constant chemical potential, $\mu$, the system is described by the grand canonical ensemble where the grand canonical partition function is given by Eqn. 2.

$$
\Xi(\mu, V, \beta)=\sum_{N=0}^{\infty} \exp (\beta \mu N) Q(N, V, \beta)
$$

In this ensemble, the probability of observing $N$ particles in the system is given by Eqn. 3 :

$$
\Pi(N ; \mu, V, \beta)=\frac{\exp (\beta \mu N) Q(N, V, \beta)}{\Xi(\mu, V, \beta)}
$$

By specifying $\mu, V$, and $T$, one can use grand canonical MC simulations to sample the probability distribution in Eqn. 3 and compute, among other things, the expected number of particles in the system.

\section{Grand canonical flat-histogram simulations}

Flat-histogram techniques operate quite differently from the standard MC approach. They seek to bias the simulation so that a collective variable, or macrostate, is sampled uniformly, i.e., all macrostates are visited with equal probability and thus have a flat probability distribution. Therefore, macrostates that have a high free energy relative to other states become 
equally probable when the biasing function has been properly determined, and an efficient method to achieve this goal was pioneered by Wang and Landau. ${ }^{10,26,27}$ When studying adsorption, a convenient macrostate variable is the number of particles in the system, $N$. Upon convergence of the WL algorithm, $\Pi(N ; \mu, V, \beta)$ is known for each macrostate $N$, which can then be re-weighted to some determine $\Pi\left(N ; \mu^{\prime}, V, \beta\right)$ at some new chemical potential $\mu^{\prime}$ by simple post-processing. ${ }^{20,24}$ Alternatively, for single-component systems one can directly solve $Q(N, V, \beta)$ as the biasing function rather than $\Pi(N ; \mu, V, \beta) .{ }^{17}$

\section{Transition matrix Monte Carlo formulation}

Typically the Wang-Landau approach builds up an initial estimate of $\Pi(N ; \mu, V, \beta)$ quickly but converges rather slowly. Transition matrix Monte Carlo (TMMC) tends to do the opposite, so a simulations often start with a WL stage which is later switched to TMMC to

accumulate the statistics that refine the exact values of the biasing function. ${ }^{13}$ The starting point for TMMC is the statement of detailed balance for Monte Carlo particle exchange moves between macrostates:

$$
\Pi(N ; \mu, V, \beta) P(N \rightarrow N+1)=\Pi(N+1 ; \mu, V, \beta) P(N+1 \rightarrow N)
$$

Here $P(N \rightarrow N+1)$ represents the probability that a proposed MC move takes the system from macrostate $N$ to macrostate $N+1$, given that the system was already in macrostate $N$. The remaining task is to determine the values of $P(N)$. This is done by constructing the collection matrix (C-matrix). Each entry in the C-matrix is updated by evaluation of the unbiased acceptance rule, $\operatorname{acc}\left(\Gamma_{\mathbf{r}^{N}} \rightarrow \Gamma_{\mathbf{r}^{N+1}}\right)$ when a swap move is proposed. For example, every time a particle addition move is proposed, the $N^{\text {th }}$ row and $(N+1)^{\text {th }}$ column is updated with the probability of accepting such a transition:

$$
C(N, N+1)=C(N, N+1)+\operatorname{acc}\left(\Gamma_{\mathbf{r}^{N}} \rightarrow \Gamma_{\mathbf{r}^{N+1}}\right)
$$


Simultaneously, the probability that such a transition is rejected is also used to update the C-matrix.

$$
C(N, N)=C(N, N)+\left[1-\operatorname{acc}\left(\Gamma_{\mathbf{r}^{N}} \rightarrow \Gamma_{\mathbf{r}^{N+1}}\right)\right]
$$

The same concept applies to updating the C-matrix for particle deletion moves, and since only single particle additions or deletions are proposed, the C-matrix is tridiagonal. The transition probability can be computed at the end of the simulation from the C-matrix via Eqn. 7.

$$
P(N \rightarrow N+1)=\frac{C(N, N+1)}{\sum_{\Delta \in\{-1,0,1\}} C(N, N+\Delta)}
$$

$P(N)$ may then be used to obtain $\Pi(N ; \mu, V, \beta)$ via Eqn. 4. Regarding the implementation of this method, the simulation can be performed at any arbitrary value of $\mu$. The macrostate probabilities can then be easily re-weighted to other values of the chemical potential to obtain $\Pi\left(N ; \mu^{\prime}, V, \beta\right)$, which is extensively detailed elsewhere. ${ }^{20,24}$ It will be useful in subsequent sections to note that Eqn. 4 can be combined with Eqns. 1 and 3 such that the calculation of $\Pi(N ; \mu, V, \beta)$ is re-expressed as a calculation of $\ln Q_{c}(N+1, V, \beta)$ in Eqn. 8.

$$
\ln Q_{c}(N+1, V, \beta)=\ln Q_{c}(N, V, \beta)-\ln [q(\beta) \exp (\beta \mu)]+\ln \left[\frac{P(N \rightarrow N+1)}{P(N+1 \rightarrow N)}\right]
$$

\section{Temperature extrapolation of the free energy landscape}

For TMMC, we expressed Eqn. 8 in terms of $\ln Q_{c}(N, V, \beta)$ to demonstrate how to most efficiently perform temperature extrapolation. The canonical partition function at some new temperature, $\beta^{\prime}$, is just the product of the kinetic and configurational partition functions at this new temperature, or $Q\left(N, V, \beta^{\prime}\right)=q\left(\beta^{\prime}\right)^{N} Q_{c}\left(N, V, \beta^{\prime}\right)$. The kinetic partition function is known exactly at this new temperature since $\ln q\left(\beta^{\prime}\right)^{N}=-3 N \ln \left(\Lambda^{\prime}\right)$. The configura- 
tional partition function is not known analytically; however, it can be estimated at this new temperature by a Taylor series expansion truncated to some order $m$, as shown in Eqn. 9 .

$$
\ln Q_{c}\left(N, V, \beta^{\prime}\right) \approx \ln Q_{c}(N, V, \beta)+\sum_{n \geq 1}^{m} \frac{1}{n !} \frac{\partial^{n} \ln Q_{c}(N, V, \beta)}{\partial \beta^{n}}\left(\beta^{\prime}-\beta\right)^{n}
$$

Since $\ln Q_{c}(N, V, \beta)$ is a cumulant generating function and related to the fluctuations in the system's total potential energy, the derivative terms in the Taylor expansion can be evaluated by simply recording the potential energy from the simulation at the original temperature, $\beta$, and computing ensemble-averaged quantities, such as:

$$
\begin{aligned}
& \frac{\partial \ln Q_{c}(N, V, \beta)}{\partial \beta}=-\langle E\rangle \\
& \frac{\partial^{2} \ln Q_{c}(N, V, \beta)}{\partial \beta^{2}}=\left\langle(E-\langle E\rangle)^{2}\right\rangle \\
& \frac{\partial^{3} \ln Q_{c}(N, V, \beta)}{\partial \beta^{3}}=-\left\langle(E-\langle E\rangle)^{3}\right\rangle \\
& \frac{\partial^{4} \ln Q_{c}(N, V, \beta)}{\partial \beta^{4}}=\left\langle(E-\langle E\rangle)^{4}\right\rangle-3\left(\left\langle(E-\langle E\rangle)^{2}\right\rangle\right)^{2}
\end{aligned}
$$

Higher order terms may also be derived. ${ }^{21}$ Using the Taylor series expansion to approximate $\ln q(\beta)$ at a different temperature would have been highly undesirable since the analytic solution is already known for any temperature. ${ }^{22}$ This has been avoided by factoring the total partition function so that the Taylor approximation is only applied to $\ln Q_{c}(N, V, \beta)$. Finally, the extent to which $\ln Q_{c}(N, V, \beta)$ can be accurately extrapolated at each of the $N$ macrostates depends on how well the moments of the potential energy distribution have converged throughout the simulation. The results section demonstrates that this extrapolation can be valid over a surprisingly large temperature range. 


\section{Extension to deformable adsorbates}

The formalism presented thus far has implicitly assumed that the particles are rigid bodies with no internal degrees of freedom. Molecules with internal degrees of freedom often require additional sampling biases to explore configurational space effectively. One such approach we focus on is Rosenbluth sampling. ${ }^{6,7}$ Additional complexities must be accounted for in order to perform temperature extrapolation on TMMC simulations of deformable molecules using this sampling scheme, which we will now illustrate.

\section{Rosenbluth sampling}

The standard, unbiased acceptance rule for particle insertions in GCMC can become prohibitively small for the simulation of deformable adsorbates, since the vast majority of randomly generated configurations would yield an extremely high internal energy arising from intramolecular potentials (bonds, bends, torsions, etc.). Rosenbluth sampling presents an efficient way to overcome this obstacle by biasing the growth of chain molecules during a MC move. ${ }^{6,8}$ In the case of flat-histogram MC, it is also necessary to take advantage of such a biasing scheme when accumulating statistics for the C-matrix. The acceptance rule for a MC particle insertion move using Rosenbluth sampling (in the notation of Ref. 1) is given as follows:

$$
\operatorname{acc}\left(\Gamma_{\mathbf{r}^{N}} \rightarrow \Gamma_{\mathbf{r}^{N+1}}\right)=\min \left[1, \frac{V q(\beta) \exp \left(\beta \mu_{B}\right)}{(N+1)} \mathcal{W}^{\operatorname{ext}}\left(\Gamma_{\mathbf{r}^{N+1}}\right)\right]
$$

where $\mathcal{W}^{\text {ext }}\left(\Gamma_{\mathbf{r}^{N+1}}\right)$ is the Rosenbluth factor of the inserted molecule and $\mu_{B}$ is the chemical potential of a reservoir of "ideal chain" molecules (see the following section for details). In Rosenbluth sampling, only the chain's external (intermolecular and non-bonded intramolecular) interactions determine its acceptance probability, while reasonable trial configurations are generated using only the intramolecular bonded interactions. An in-depth discussion of this biasing technique and the algorithm for computing $\mathcal{W}^{e x t}\left(\Gamma_{\mathbf{r}^{N+1}}\right)$ is presented extensively 
in Ref. 1 and elsewhere. ${ }^{28,29}$

\section{Ideal chain partition function}

In order to extrapolate the thermodynamic observables of a system of chain molecules simulated using Rosenbluth sampling, ${ }^{1}$ the dependence of the ideal chain partition function on temperature must also be accounted for. An ideal chain is considered to have only intramolecular bonded interactions, and so its partition function represents the integration over each bonded degree of freedom in the molecule. We focus on propane $\left(\mathrm{C}_{3} \mathrm{H}_{8}\right)$ for the remainder of this work which has two bonds and one angle potential such that the ideal chain partition function becomes:

$$
Q_{I C, c}(\beta)=\int d r_{1} \int d r_{2} \int d \theta_{1} \exp \left(-\beta\left(E_{\text {bond }}\left(r_{1}\right)+E_{\text {bond }}\left(r_{2}\right)+E_{\text {angle }}\left(\theta_{1}\right)\right)\right.
$$

$Q_{I C, c}(\beta)$ represents the configurational component of the partition function which has been separated from the integration of kinetic degrees of freedom, $q(\beta)$, such that the total ideal chain partition function becomes $Q_{I C}(\beta)=Q_{I C, c}(\beta) q(\beta)$. The default TraPPE description ${ }^{30}$ of propane in the RASPA package ${ }^{31}$ was used to evaluate the $E_{\text {bond }}$ and $E_{\text {angle }}$ terms. Such an integral can be easily handled directly, and the value of the ideal chain partition function can be found by numerical integration for any temperature. However, for larger adsorbates with many internal degrees of freedom, the ideal chain partition may be a much more difficult integral to evaluate. A simulation-based alternative to handle these cases is discussed in the Supporting Information (SI).

\section{Chemical potential of deformable adsorbates}

To perform temperature extrapolation of TMMC simulations with Rosenbluth sampling, it is critical to see how the chemical potential of a reservoir of ideal chain molecules depends on the ideal chain partition function. First, consider a reservoir of rigid ideal gas particles: 


$$
\beta \mu=\beta \mu^{o}+\ln (\rho)
$$

Here $\rho$ is the fluid density, which in the case of an ideal gas, is equal to $\beta \mathcal{P}$ where $\mathcal{P}$ is the pressure. For real fluids, we can account for the non-ideality of the reservoir by replacing $\mathcal{P}$ with the fugacity, $f=\phi \mathcal{P}$, after obtaining the fugacity coefficient, $\phi$, from an equation of state. The reference state chemical potential, $\beta \mu^{o}=-\ln q(\beta)$, derives from the canonical partition function of a single ideal gas particle (which for a rigid particle is just the integration over its kinetic degrees of freedom).

If the reservoir consists of ideal chains, the chemical potential of such a fluid takes a similar form as Eqn. 13, but the reference state no longer accounts only for an integration over the kinetic degrees of freedom. Now the reference state must also account for the fact that the ideal chain has some potential energy interactions associated with its bonded internal degrees of freedom. Unlike the case of an ideal gas of rigid molecules, now there are interactions that cannot be neglected in the limit of zero density. Thus, the reference state chemical potential is shifted by the temperature dependent ideal chain configurational partition function to give the chemical potential of an ideal chain, $\mu_{I C}$, in Eqn. 14. 1,5

$$
\beta \mu_{I C}=-\ln Q_{I C}(\beta)=-\ln q(\beta)-\ln Q_{I C, c}(\beta)
$$

The chemical potential of a reservoir of these ideal chain molecules, $\mu_{B}$, is similar to Eqn. 13 where the reference state $\beta \mu^{o}$ has been replaced by $\beta \mu_{I C}$.

$$
\beta \mu_{B}=\left[-\ln q(\beta)-\ln Q_{I C, c}(\beta)+\ln (\beta \phi \mathcal{P})\right]+C
$$

Note we have introduced a temperature-dependent shift to the reservoir chemical potential in Eqn. 15, where $C=-\ln \left\langle\mathcal{W}_{I G}^{e x t}(\beta)\right\rangle$. Known as the ideal gas Rosenbluth weight, $\mathcal{W}_{I G}^{e x t}$ accounts for when non-bonded intramolecular interactions can contribute to an isolated chain's partition function due to the molecule's size (e.g., 1-5 pair interactions). It's inclusion 
in Eqn. 15 is derived in detail elsewhere. ${ }^{5,28,29}$ By using propane as our flexible adsorbate we can presently ignore this possibility $\left(\left\langle\mathcal{W}_{I G}^{\text {ext }}(\beta)\right\rangle=1\right)$, which allows us to proceed with a slightly more concise derivation of the temperature extrapolation in subsequent sections. As in the case of rigid adsorbates, we can account for the non-ideality of the reservoir phase by replacing $\mathcal{P}$ with $f$ and obtaining the fugacity coefficient from an equation of state. Alternatively, direct simulations of the bulk fluid can provide this information if a reliable equation of state is not readily available. ${ }^{23}$

\section{Constructing and Extrapolating the Macrostate Distribution}

The TMMC formulation for chain molecules now becomes slightly more complex when using Rosenbluth sampling. This is because Rosenbluth sampling for particle insertions only yields the excess chemical potential relative to the ideal chain, a topic which we expand upon in the SI (this discussion closely relates to the formalism presented in Ref. 32 where the excess chemical potential of the ideal gas reference state of chain molecules was also computed in the context of a TMMC study). Thus, in order to properly compute the value of the total configurational partition function, one must add the contribution from the ideal chain when calculating $\ln Q_{c}(N, V, \beta)$ at each successive $N$ macrostate, as shown in Eqn. 16.

$\ln Q_{c}(N+1, V, \beta)=\ln Q_{I C, c}(\beta)+\ln Q_{c}(N, V, \beta)-\ln \left[q(\beta) \exp \left(\beta \mu_{B}\right)\right]+\ln \left[\frac{P(N \rightarrow N+1)}{P(N+1 \rightarrow N)}\right]$

Thus, from a TMMC simulation at a chosen $\beta$, we may construct $\ln Q_{c}(N, V, \beta)$ using the transition probabilities, $P$, computed from the C-matrix; what remains is to then predict the macrostate distribution, $\ln \Pi\left(N, V, \beta^{\prime}\right)$, at arbitrary $\beta^{\prime}$ via extrapolation. Following Eqn. 3, assuming the fugacity coefficient is known from an equation of state, we obtain: 


$$
\begin{aligned}
\ln \Pi\left(N ; \mu, V, \beta^{\prime}\right) & \sim \beta^{\prime} \mu N+\ln Q\left(N, V, \beta^{\prime}\right) \\
& \sim\left[\ln \left(\beta^{\prime} \phi^{\prime} \mathcal{P}\right)-\ln q\left(\beta^{\prime}\right)-\ln Q_{I C, c}\left(\beta^{\prime}\right)\right] N+\ln Q_{c}\left(N, V, \beta^{\prime}\right)+\ln Q_{k}\left(N, \beta^{\prime}\right) \\
& \sim\left[\ln \left(\beta^{\prime} \phi^{\prime} \mathcal{P}\right)-\ln Q_{I C, c}\left(\beta^{\prime}\right)\right] N+\ln Q_{c}\left(N, V, \beta^{\prime}\right) \\
& \sim\left[\ln \left(\beta^{\prime} \phi^{\prime} \mathcal{P}\right)-\ln Q_{I C, c}\left(\beta^{\prime}\right)\right] N+\ln Q_{c}(N, V, \beta)+ \\
& \quad \sum_{n \geq 1}^{m} \frac{1}{n !} \frac{\partial^{n} \ln Q_{c}(N, V, \beta)}{\partial \beta^{n}}\left(\beta^{\prime}-\beta\right)^{n} \\
& \sim N \ln \left(\beta^{\prime} \phi^{\prime} \mathcal{P}\right)+\left[\ln Q_{c}(N, V, \beta)-N \ln Q_{I C, c}\left(\beta^{\prime}\right)\right]+ \\
& \sum_{n \geq 1}^{m} \frac{1}{n !} \frac{\partial^{n} \ln Q_{c}(N, V, \beta)}{\partial \beta^{n}}\left(\beta^{\prime}-\beta\right)^{n}
\end{aligned}
$$

Following Eqn. 16, observe that $\ln Q_{c}(N, V, \beta)$ in the macrostate probabilities implicitly contains a contribution of $N \times \ln Q_{I C, c}(\beta)$ since it is constructed iteratively from $P(N)$ starting from the $N=0$ state. Thus, the bracketed term in the final line explicitly shows that differences between the ideal chain partition functions at the simulation temperature and the temperature being extrapolated to contribute to the macrostate probabilities at a chosen $\mathcal{P}$ and $\phi$. Note that in Eqn. 17, $Q_{I C, c}(\beta)=1$ for rigid adsorbates. By summing the terms in Eqn. 17, we obtain an estimate of the grand canonical partition function (Eqn. 2), which serves to normalize the macrostate probabilities. Just as in the case of adsorbates with no internal degrees of freedom, one uses the moments of the total potential energy distribution (which now included all bonded and non-bonded energies) to evaluate terms in the Taylor series of $\ln Q_{c}(N, V, \beta)$.

\section{Extraction of thermodynamic properties}

Temperature extrapolation of $\ln Q(N, V, \beta)$ yields continuous thermodynamic properties that cannot readily be obtained from standard GCMC simulations. For example, isotherms and 
isosteric heats of adsorption can be computed for any combination of reservoir $(T, \mathcal{P})$ state points via a computationally cheap post-processing of the $\ln Q(N, V, \beta)$ data. These are typically the first two important quantities considered when evaluating a material's potential as an adsorbent.

\section{Isotherm prediction}

The adsorption isotherm at a given temperature, $\beta^{\prime}$, and chemical potential, $\mu^{\prime}$, can be resolved by computing the expectation number of particles in the system via Eqn. 18 for a range of pressures (or equivalently, chemical potentials).

$$
\langle N\rangle_{\mu^{\prime}, V, \beta^{\prime}}=\sum_{N} N \cdot \Pi\left(N ; \mu^{\prime}, V, \beta^{\prime}\right)
$$

The ability to calculate this quantity at any $\mu^{\prime}$ (via re-weighting) and $\beta^{\prime}$ (subsequent extrapolation) from simulation data obtained at some different, single $(\mu, \beta)$ point results in significantly more predictive power than running simple GCMC simulations for individual $(\mu, \beta)$ state points.

\section{Enthalpy of adsorption}

Another important thermodynamic quantity for predicting an adsorbent material's utility is the isosteric heat (or enthalpy of adsorption). The isosteric heat of adsorption measures how much enthalpy is released when an additional molecule is adsorbed and plays an important role in calculating how much energy is required when cycling between adsorption and desorption conditions. The isosteric heat of adsorption can be determined from several statistical mechanics approaches. Since the standard energy-particle fluctuation method can suffer from poor statistical convergence, Vlugt et al. proposed determining the isosteric heat from a se-

ries of NVT simulations with Widom insertions (which just requires a simple post-processing step of our TMMC simulation data). ${ }^{33}$ In this work, however, we chose to calculate the isosteric heat using the Clausius-Clapeyron equation. Isotherms can be obtained for arbitrarily 
small spacing between different $T$ and $P$ state points via the aforementioned post-processing and temperature extrapolation, therefore the derivative in the Clausius-Clapeyron equation (Eqn. 19) can be numerically evaluated.

$$
q_{s t}(N, T)=-R T^{2}\left(\frac{\partial \ln \mathcal{P}}{\partial T}\right)_{N}
$$

Thus, $q_{s t}$ as a function of $N$ and $T$ is known over the temperature range at which the temperature extrapolation is valid and the total enthalpy change between two states $\left(N_{1}\right.$, $\left.T_{1}\right)$ and $\left(N_{2}, T_{2}\right)$ is easily calculated by integrating Eqn. $19 .{ }^{34}$ As noted in Ref. 34 one can replace $\mathcal{P}$ with the fugacity to account for the non-ideality of the vapor phase. While the isosteric heat often has only a weak dependence on temperature, it is interesting that this dependence can be explicitly computed via this method. In systems where the heat of adsorption has a strong dependence on temperature, the use of flat histogram simulations to compute isosteric heats via this method provides a highly attractive alternative to GCMC.

\section{Computational details}

\section{Adsorbate/adsorbent system}

Results in this work focus on the performance of the flat-histogram simulation of singlecomponent adsorbate thermodynamic properties of $\mathrm{CH}_{4}, \mathrm{CO}_{2}$, and $\mathrm{C}_{3} \mathrm{H}_{8}$ in MOF-950. MOF950, visualized in Fig. 1, was chosen as a model system because it was recently shown to have a high working capacity for methane. ${ }^{35} \mathrm{CH}_{4}$ and $\mathrm{CO}_{2}$ were modeled as rigid adsorbates with the united-atom TraPPE and EPM2 forcefields, respectively. ${ }^{30,36} \mathrm{C}_{3} \mathrm{H}_{8}$ was modeled as a flexible adsorbate, also with the united-atom TraPPE forcefield. The molecule definition files for these adsorbates are provided in the standard RASPA installation. The frame-

work was modeled as rigid, and the Universal Force Field parameters were adopted for the MOF atoms. ${ }^{37}$ Interaction parameters between the MOF and adsorbate were determined 
by Lorentz-Berthelot combining rules. The MOF-950 crystallographic information file is included in the SI.

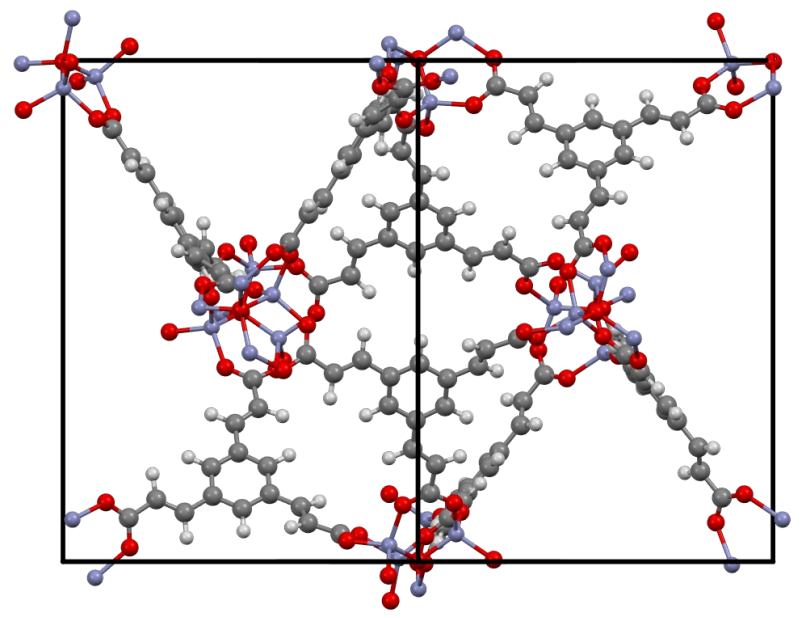

Figure 1: Visual representation of the MOF-950 framework with the viewing plane parallel to the 011 face.

\section{Transition matrix MC implementation}

Before using TMMC, one typically wants to obtain a good initial estimate of the macrostate probabilities by quickly exploring the entire macrostate space via the WL algorithm. While TMMC can technically operate with any initial biasing array, it will more efficiently explore macrostate space if the initial biasing array approximates the true macrostate probabilities which are roughly determined from a short WL run. In practice, macrostate space is also distributed in discrete chunks for parallel computation. The results of the individual simulations are then stitched back together to obtain the free energy across the entire macrostate space. Significant previous work has focused on determining the optimal way to distribute the macrostate blocks to each individual flat-histogram simulation. ${ }^{38,39}$ In this work we alternatively solve the macrostate probabilities, or $\ln Q(N, V, \beta)$, by running many simulations in the canonical ensemble for each value of $N$ while performing Widom insertions. ${ }^{1}$ By imposing constant $N$ simulations, we can artificially make the "observation" of each macrostate 
equally likely by performing $N=1 \ldots N_{\max }$ different NVT simulations. Therefore, it is not necessary to ever construct or update a biasing function to make the sampling probability distribution flat as in WL/TMMC for effective macrostate exploration; however, we can still use the principles of TMMC to obtain the transition probabilities via Widom insertions and deletions. This is formally equivalent to taking a window size of 1 , as has been already performed in Ref. 16. In other words, performing a flat-histogram simulation with a window size of 1 reduces the flat-histogram simulation to an $N_{\max }$ number of NVT simulations where the C-matrix statistics are consequently populated by Widom insertions and deletions. For simplicity, we henceforth refer to a TMMC simulation with window size of 1 as an NVT $+\mathrm{W}$ simulation. It should be noted that this ghost insertion approach in individual canonical ensemble simulations works well for supercritical fluids, which are the conditions of interest for high-throughput screening of materials' separation performance for light gases, but we have not explored its efficacy with temperature extrapolation in subcritical regimes where phase coexistence generates a bimodal macrostate distribution.

\section{Simulation details}

All simulations were performed with the RASPA code. ${ }^{31} \mathrm{NVT}+\mathrm{W}$ simulations were performed to obtain the data necessary to generate temperature extrapolated isotherms and isosteric heats, and the accuracy of these temperature extrapolated thermodynamic properties are compared to standard GCMC simulations. For GCMC simulations, $5 \times 10^{4}$ cycles were utilized for both equilibration and production. Each NVT + W simulation was equilibrated for $5 \times 10^{3}$ cycles, and $4 \times 10^{3}$ cycles were used to accumulate the statistics necessary to construct the C-matrix, where translation/rotation/regrow/Widom moves were proposed in a ratio of $1 / 1 / 1 / 2$. Cycles were defined such that this led to a total of at least $3.2 \times 10^{4} \times N$ Widom insertions and deletions acquired for each NVT simulation. For each adsorbate, the Peng-Robinson equation of state was used to compute the fugacity coefficient of the reservoir fluid for a given temperature and pressure, ${ }^{40}$ under the assumption that it faithfully captures 
the bulk behavior of the simulation model. The input files required to run the simulations are provided in the SI.

\section{Results}

\section{Isotherms of $\mathrm{CH}_{4}, \mathrm{CO}_{2}$, and $\mathrm{C}_{3} \mathrm{H}_{8}$ in MOF-950}

Fig. 2 shows the isotherms computed from $\mathrm{NVT}+\mathrm{W}$ and GCMC simulations for rigid $\mathrm{CH}_{4}$ and $\mathrm{CO}_{2}$ and deformable $\mathrm{C}_{3} \mathrm{H}_{8}$ in MOF-950. In this figure, open circles represent GCMC predicted loadings, while dashed lines represent the calculation of an isotherm at the colorcoded temperature from an $\mathrm{NVT}+\mathrm{W}$ simulation at that same temperature. Solid lines represent the temperature extrapolated isotherms from the $\mathrm{NVT}+\mathrm{W}$ simulation data originally obtained at one single temperature, $T_{\text {sim }}$, using up to third order terms $(m=3)$ in Eqn. 9. The temperature extrapolated isotherms for $\mathrm{CH}_{4}$ (Fig. 2a-b), $\mathrm{CO}_{2}$ (Fig. 2c-d) and $\mathrm{C}_{3} \mathrm{H}_{8}$ (Fig. 2e-f) are generated from $\mathrm{NVT}+\mathrm{W}$ simulations at $T_{\text {sim }}=270 \mathrm{~K}, T_{\text {sim }}=300 \mathrm{~K}$ and $T_{\text {sim }}=400 \mathrm{~K}$, respectively. In all cases the GCMC isotherms match the temperature extrapolated isotherms over an extremely large temperature extrapolation range, and the $\log$-log representation of the data shows that the Henry coefficients match exactly. Interestingly, the temperature extrapolation for $\mathrm{CO}_{2}$ perfectly capture the non-Langmuirian shape (low pressure inflection) of the adsorption isotherms for all temperatures.

To evaluate a material's potential as an adsorbent, one is often interested in calculating working capacity, or the difference in uptake between the adsorption and desorption temperature and pressure. Since we can evaluate the number of particles adsorbed (Eqn. 18) for any given number of temperatures and pressures by post-processing of the $\ln Q_{c}(N, V, \beta)$ simulation data, the working capacity can be easily calculated via Eqn. 20.

$$
n_{w c}=\langle N\rangle_{\mu_{2} V \beta_{2}}-\langle N\rangle_{\mu_{1} V \beta_{1}}
$$



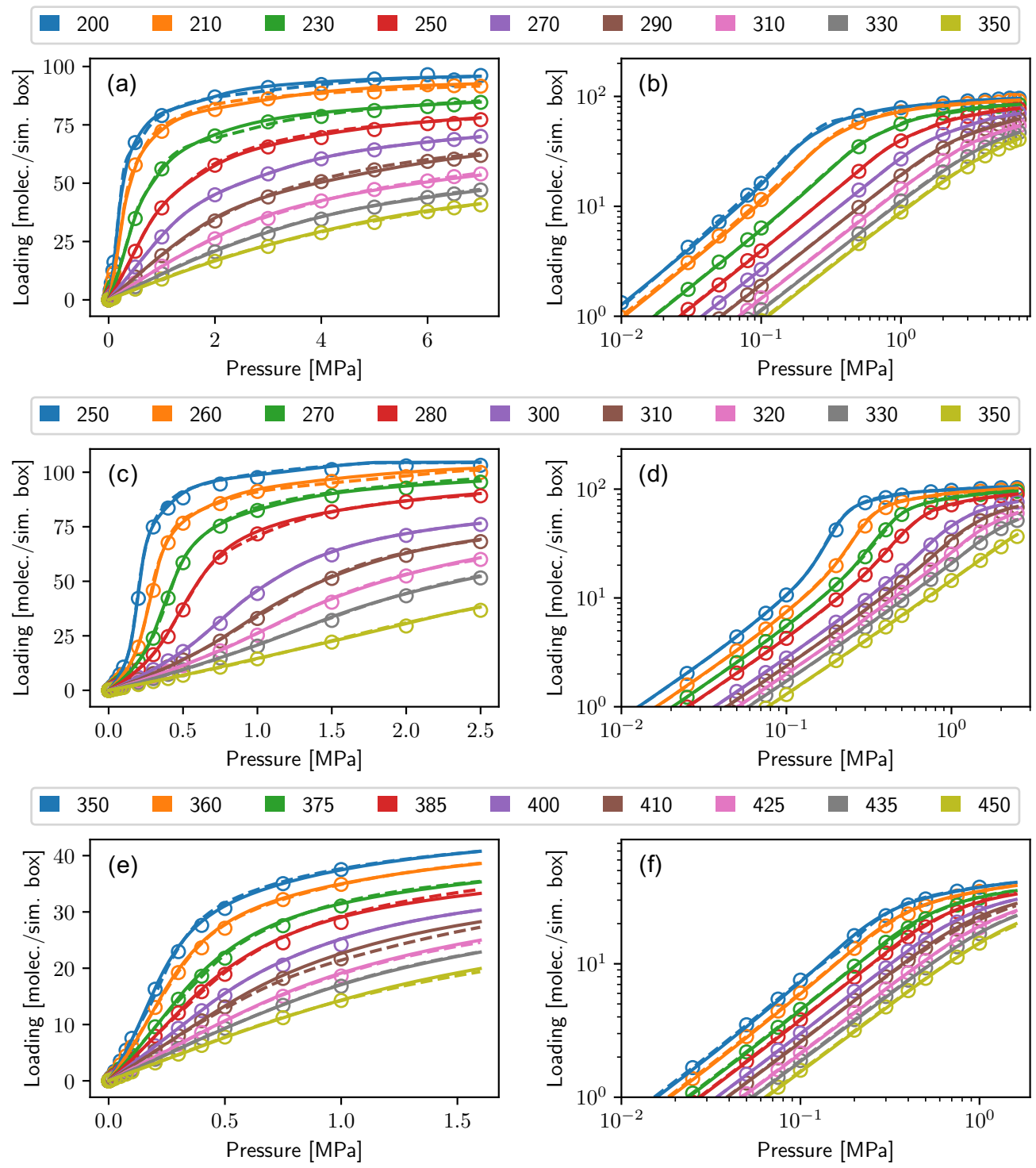

Figure 2: $\mathrm{CH}_{4}(\mathrm{a}-\mathrm{b}), \mathrm{CO}_{2}(\mathrm{c}-\mathrm{d})$, and $\mathrm{C}_{3} \mathrm{H}_{8}$ (e-f) isotherms are shown for MOF-950. (b), (d), and (f) are plotted on log-log axes to show the agreement between all calculations in the low-pressure, Henry's Law regime. Open circles represent GCMC simulations, dashed lines represent $\mathrm{NVT}+\mathrm{W}$ calculated isotherms from a simulation at the specified temperature, and solid lines represent temperature extrapolated isotherms from an NVT $+\mathrm{W}$ simulation originally performed at a single temperature $\left(T_{\text {sim }}=270 \mathrm{~K}\right.$ for $\mathrm{CH}_{4}, T_{\text {sim }}=300 \mathrm{~K}$ for $\mathrm{CO}_{2}$, and $T_{\text {sim }}=400 \mathrm{~K}$ for $\mathrm{C}_{3} \mathrm{H}_{8}$ ).

In a combined temperature and pressure swing adsorption ( $\mathrm{T} / \mathrm{PSA}$ ) process, both temperature and pressure change between the adsorption and desorption conditions; however, an NVT $+\mathrm{W}$ simulation at only one temperature is needed to obtain any $\mathrm{T} / \mathrm{PSA}$ working 
capacity, assuming that the Taylor series is sufficient to approximate the temperature dependence of $\ln Q_{c}(N, V, \beta)$. From an engineering perspective, this is a marked advantage of $\mathrm{NVT}+\mathrm{W}$ simulations over unbiased GCMC since we can numerically compute nearly continuous working capacities as a function of $\left(\mu_{2}, \beta_{2}, \mu_{1}, \beta_{1}\right)$ state points and use this data as input into process simulation software.

\section{Isosteric heats of $\mathrm{CH}_{4}, \mathrm{CO}_{2}$, and $\mathrm{C}_{3} \mathrm{H}_{8}$ in MOF-950}

The isosteric heat of adsorption was computed for each adsorbate in MOF-950. Using the temperature extrapolated isotherms where $\langle N\rangle_{\mu V T}$ was generated on a grid of $\Delta T=2 \mathrm{~K}$ and $\Delta P=250 \mathrm{~Pa}$ intervals, the derivative in the Clausius-Clapeyron equation was numerically evaluated. Figure 3 shows that the heat of adsorption predicted via this methodology produces the same results as GCMC simulations which use the particle-energy fluctuation method at a fixed temperature. ${ }^{33}$

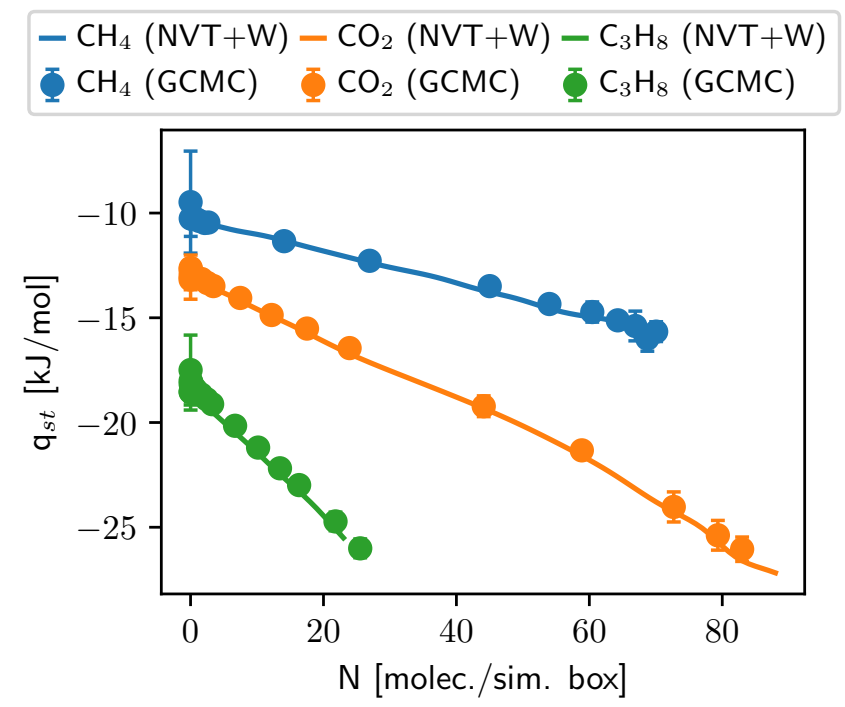

Figure 3: The isosteric heats of adsorption are plotted as a function of loading for $\mathrm{CH}_{4}, \mathrm{CO}_{2}$, and $\mathrm{C}_{3} \mathrm{H}_{8}$ at temperatures of $270 \mathrm{~K}, 300 \mathrm{~K}$, and $400 \mathrm{~K}$, respectively.

However, an added benefit can be achieved by using the flat-histogram technique to solve the isosteric heats. Fig. 4 shows the isosteric heat of adsorption, $q_{s t}(N, T)$, plotted as a 
continuous function of temperature and loading for $\mathrm{CO}_{2}$ in MOF-950. This data is only accessible due to the evaluation of the Clausius-Clapeyron equation on the aforementioned grid of $\langle N\rangle_{\mu V T}$ values, which would otherwise take a large number of GCMC simulations at many different temperatures and pressures to obtain. As expected the temperature dependence of the isosteric heat is small, but interestingly the temperature extrapolated $\mathrm{NVT}+\mathrm{W}$ data combined with the Clausius-Clapeyron analysis quantifies the subtle dependence of $q_{s t}$ on temperature. Another advantage of obtaining the data visualized in Fig. 4 is that the total change in enthalpy between any two states (termed the enthalpy of immersion in Ref. 34) can be determined by numerical integration of the isosteric heat values along the path between the two states. Also note that, since we have determined $\ln Q(N, V, \beta)$, one can also compute the entropy of adsorption as a function of loading via the Helmholtz free energy, $F(N, V, \beta)=-k_{B} T \ln Q(N, V, \beta)$.

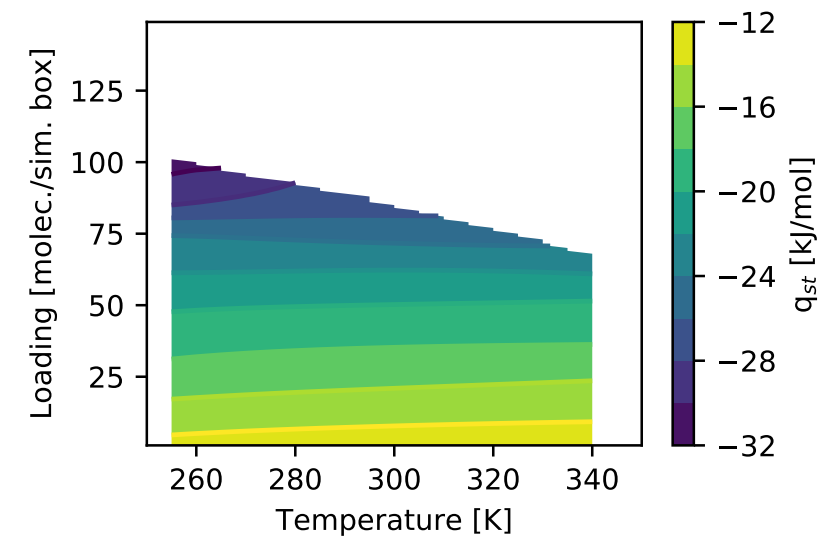

Figure 4: The full isosteric heat diagram, $q_{s t}(N, T)$, is shown for $\mathrm{CO}_{2}$ in MOF-950.

\section{Statistical performance of temperature extrapolation}

The temperature range over which the macrostate distribution can be extrapolated depends on the order at which the Taylor series approximation is truncated and how well the moments of the potential energy distribution have converged. To show the accuracy of the temperature extrapolation, we performed $\mathrm{NVT}+\mathrm{W}$ simulations for a large number of $\beta$ val- 
ues to compute $\ln Q_{\text {true }}(N, V, \beta)$. Next we took the values from one particular temperature, $\beta_{\text {sim }}$, and extrapolated them to every other temperature, denoted by $\ln Q_{\text {extrap }, \beta_{s i m}}(N, V, \beta)$. The relative error (RE) can be computed for each $N$ macrostates and each extrapolation temperature $\beta$ by Eqn. 21, and the results of this evaluation for all three adsorbates in MOF950 are shown in Fig. 5. These are encouraging results for our ability to effectively screen continuous thermodynamic adsorption properties of light gases in the supercritical regime since the range over which the macrostates can be extrapolated is quite large. For the more difficult cases of deeply subcritical fluid regimes, future investigation on the efficacy of this temperature extrapolation is warranted.

$$
R E\left(\beta, \beta_{\text {sim }}\right)=100 \times \frac{\ln Q_{\text {true }}(N, V, \beta)-\ln Q_{\text {extrap }, \beta_{\text {sim }}}(N, V, \beta)}{\ln Q_{\text {true }}(N, V, \beta)}
$$

Two characteristic decreases in performance can be observed from Fig. 5. First, the RE performance decreases for NVT states corresponding to very high particle densities, most notably with $\mathrm{CO}_{2}$. However, the regions of highest percent error for $\mathrm{CH}_{4}$ and $\mathrm{CO}_{2}(|R E|>$ $0.5 \%)$ correspond to states with density greater than the saturation loading as can be seen from the isotherm analysis in Fig. 2. It is important to choose $N_{\max }$ greater than the one's preliminary estimate of the saturation loading to avoid missing non-zero contributions at states that contribute significantly to the system's average properties, but the poor sampling well above the actual saturation loading has little impact on the results since they contribute negligibly (have sufficiently low value of $\Pi(N)$ ) for any relevant temperature and pressure values.

The more important characteristic performance decrease arises at the extremes of the temperature extrapolation range when a $1^{\text {st }}$ order Taylor approximation is used, i.e., $m=1$ in Eqn. 9. This suggests using a $1^{\text {st }}$ order Taylor approximation is not sufficient to capture the curvature of $\ln Q(N, V, \beta)$ as a function of $\beta$ as one might expect when extrapolating over a large range of $\Delta \beta$. Interestingly, the accuracy of the $m \geq 2$ extrapolations are essentially identical, indicating that the $m \geq 3$ order terms contribute negligibly to the expansion of 

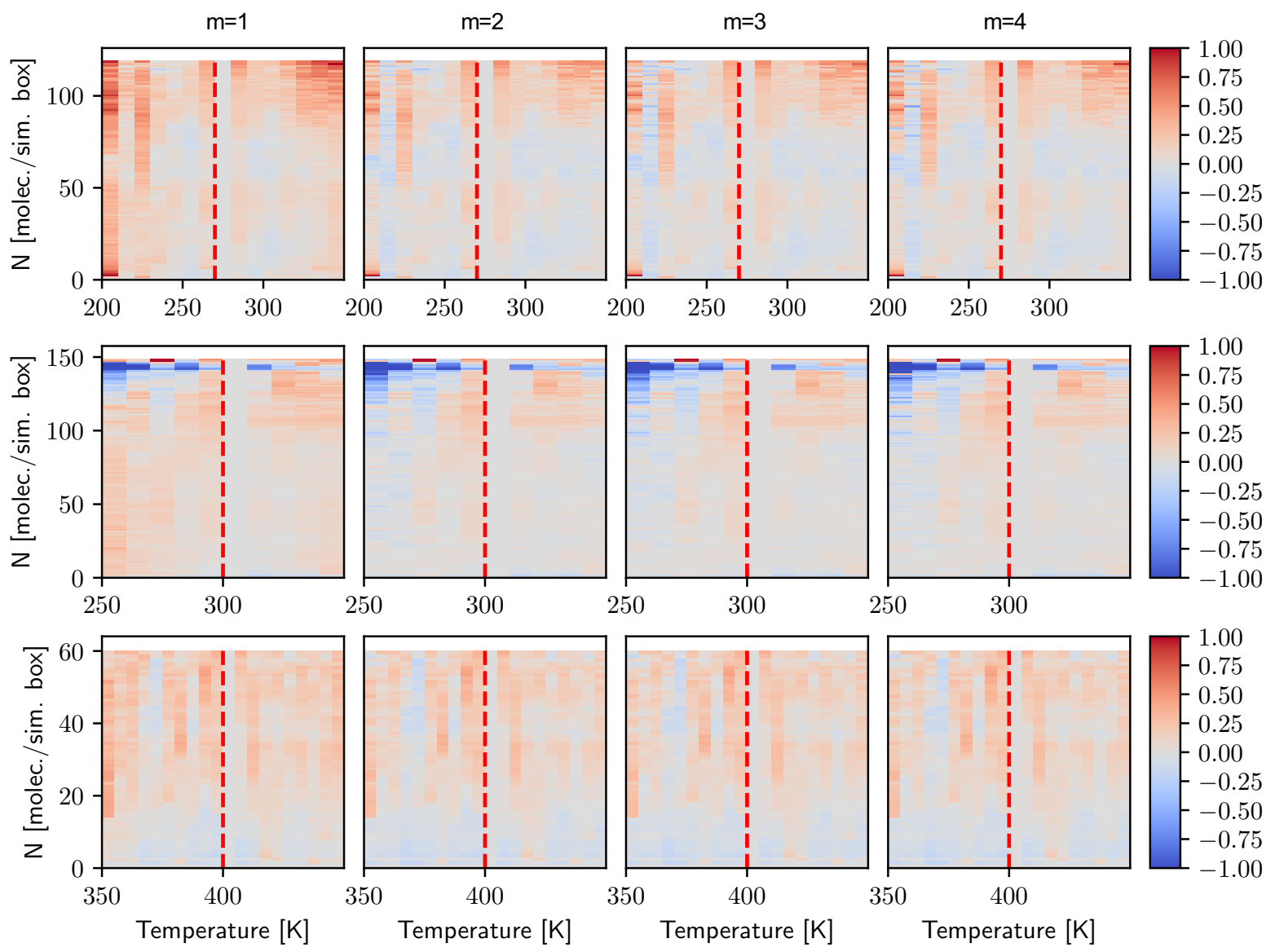

Figure 5: The first, second, and third rows of data correspond to the RE of temperature extrapolation for $\mathrm{CH}_{4}, \mathrm{CO}_{2}$, and $\mathrm{C}_{3} \mathrm{H}_{8}$, respectively. The data was extrapolated from the simulation temperatures denoted by red lines which are located at $270 \mathrm{~K}, 300 \mathrm{~K}$, and $400 \mathrm{~K}$ for for $\mathrm{CH}_{4}, \mathrm{CO}_{2}$, and $\mathrm{C}_{3} \mathrm{H}_{8}$, respectively. Each column represents the Taylor approximation with largest order $m$ (Eqn. 9). Finally, the color code is the RE of the extrapolation estimate, given by Eqn. 21.

$\ln Q(N, V, \beta)$ for these systems at these temperatures. For most of the extrapolation space, the error is within $\pm 0.5 \%$ which leads to excellent prediction of thermodynamic properties across the entire temperature range.

\section{Discussion}

First, we highlight some of the significant advantages of these flat-histogram simulations over GCMC. Most importantly, flat-histogram simulations combined with temperature ex- 
trapolation yield the macrostate distribution as a function of adsorbed particle number over a large range of temperatures. Hence, thermodynamic observables can be predicted for a broad range of conditions from a computationally cheap post-processing of simulation data obtained at a single temperature for both rigid and, as we have demonstrated, deformable adsorbates. The temperature extrapolation was not only accurate in the tested range of $\Delta T=100-150 \mathrm{~K}$, but could also be achieved with only low order moments $(m<3$ in Eqn. 9) of the potential energy distribution. This is highly encouraging as it indicates that applying this technique to study light gas adsorption under relevant supercritical $(T, P)$ process conditions does not need computationally demanding convergence of higher order moments because the curvature of the partition function is sufficiently small and can be accurately described by a low order Taylor expansion.

Since this post-processing of simulation data can be performed for arbitrarily small differences in state points, this is a powerful technique if the thermodynamic properties of an adsorbent needs to be obtained for a continuous range of temperatures and pressures, e.g., as input for process optimization software. Consider in Fig. 2a that there are approximately 100 GCMC state points plotted, yet only approximately 100 total NVT + W simulations were required to generate significantly more useful data. An arbitrarily large number of GCMC simulations would be required to interpolate the expected number of particles as a function of temperature and pressure with the same accuracy as these flat-histogram methods. In this way, flat-histogram simulations can be vastly more efficient, depending on the goal of the simulation. Also, accumulating statistics at high density states becomes more efficient with the NVT + W approach since constant N simulations are imposed for high density macrostates and the thermodynamic properties of the system are extracted from Widom insertions. In contrast, equilibrating a GCMC simulation at an extremely high density can be slow to converge due to infrequent acceptance of the particle insertion moves. The major drawback of these flat-histogram methods compared to GCMC becomes evident if one only needs to compute $\langle N\rangle_{\mu, V, \beta}$ at a very limited number of state points. 
Finally, we comment on the choice of using the NVT + W scheme, i.e., a TMMC scheme with a window size of 1 , rather than a traditional binning scheme where each bin contains a range of macrostates. While optimal binning size is an important technical consideration, we chose to run TMMC in the NVT + W scheme to simplify several implementation details. The fundamental difference between the two implementations is that NVT $+\mathrm{W}$ simply uses Widom insertions to compute transition probabilities and uses many canonical ensemble simulations to quickly build up the initial estimate of the macrostate probabilities. WL/TMMC methods often require at least an initial pass with the WL algorithm to obtain a reasonably good starting estimate for the macrostate probabilities, enabling a simulation to visit high free energy states. Therefore no implementation of WL is necessary in a MC package when generating NVT $+\mathrm{W}$ data. Performing a traditional WL/TMMC simulation requires performing C-matrix updates on the fly, implementing biased acceptance rules, and updating

biasing arrays, all of which has indeed been implemented before in freely available packages. ${ }^{41}$ In the case of $\mathrm{NVT}+\mathrm{W}$, the $\mathrm{MC}$ code simply needs to write the unbiased acceptance criteria to disk, and all macrostate probability (TMMC) calculations can be done in post-processing. This may be useful to researchers working with multiple different MC codes for which there are no implemented WL/TMMC functionalities. A minor disadvantage of NVT $+\mathrm{W}$ is that additional simulation time is spent equilibrating each individual NVT simulation, an issue not experienced with WL/TMMC. Further investigation is also required before utilizing this scheme in deeply subcritical, phase coexistence regimes.

\section{Conclusions}

We have outlined the practical utility of flat-histogram Monte Carlo simulations that, when combined with temperature extrapolation, provide significantly more thermodynamic information regarding adsorption in nanoporous materials than traditional GCMC simulations. The formalism for the temperature extrapolation of macrostate probabilities was extended 
for the first time to handle molecules with intramolecular degrees of freedom simulated using Rosenbluth sampling. This led to successful application of the method on both rigid and deformable adsorbates. Consequently, the working capacity and working enthalpy for a T/PSA process over an extremely wide range of conditions can also be ascertained from a simulation at only one temperature. Thus, the results presented here demonstrate the potential of these simulation techniques for in silico identification and study of high-performing adsorbents for realistic T/PSA processes. Mechanistically, the TMMC simulations were executed using a window size of 1 such that the problem reduces to a series of NVT simulations with Widom insertions used to compute the transition probabilities.

If the simulations community moves towards flat-histogram techniques, a material's performance can immediately be re-calculated when specified process conditions change. Rather than repeating a MC simulation at the new conditions, this can be achieved by simply reprocessing the free energy landscape from our original simulation. In other words we significantly increase the recyclability of our simulation results by performing flat-histogram MC simulations. Similar to the way that databases are currently being developed to store and benchmark results in computational material science, ${ }^{42,43}$ developing databases of free energy landscapes for different pure components or mixtures will provide more robust data as we continue to screen potential adsorbents for separations and storage applications. There also remain exciting opportunities to extend these methods to adsorption in nanoporous materials involving mixtures, for which a theoretical framework has already been developed. ${ }^{16,21}$ Upon further method development and application of more advanced sampling techniques to multi-component mixtures, these methods could lead to numerical, "model-free" optimization of the operating conditions that minimize the parasitic energy of separations in porous materials. $^{44,45}$ 


\section{Acknowledgement}

M.W. and B.S. were supported by the Center for Gas Separations Relevant to Clean Energy Technologies, an Energy Frontier Research Center funded by the DOE, Office of Science, Office of Basic Energy Sciences under award DE-SC0001015.

\section{Supporting Information Available}

The Supporting Information document provides additional discussion on temperature extrapolation of the ideal chain partition function. Supporting simulation files and crystallographic structure files are provided.

\section{References}

(1) Frenkel, D.; Smit, B. Understanding Molecular Simulations; Elsevier: San Diego, 2002.

(2) Panagiotopoulos, A. Z. Direct determination of phase coexistence properties of fluids by Monte Carlo simulation in a new ensemble. Mol. Phys. 1987, 61, 813-826.

(3) Siepmann, J. I.; Karaborni, S.; Smit, B. Simulating the critical behaviour of complex fluids. Nature 1993, 365, 330-332.

(4) Snurr, R. Q.; June, R. L.; Bell, A. T.; Theodorou, D. N. Molecular Simulations of Methane Adsorption in Silicalite. Mol. Simul. 1991, 8, 73-92.

(5) Smit, B. Grand canonical Monte Carlo simulations of chain molecules: adsorption isotherms of alkanes in zeolites. Mol. Phys. 1995, 85, 153-172.

(6) Rosenbluth, M. N.; Rosenbluth, A. W. Monte Carlo Calculation of the Average Extension of Molecular Chains. J. Chem. Phys. 1955, 23, 356-359. 
(7) Siepmann, J. I.; Frenkel, D. Configurational bias Monte Carlo: a new sampling scheme for flexible chains. Mol. Phys. 1992, 75, 59-70.

(8) Frenkel, D.; Mooji, G.; Smit, B. Novel scheme to study structural and thermal properties of continuously deformable molecules. J. Phys. Condens. Matter 1992, 4, 30533076.

(9) Shi, W.; Maginn, E. Continuous Fractional Component Monte Carlo: An Adaptive Biasing Method for Open System Atomistic Simulations. J. Chem. Theory Comput. 2007, 3, 1451-1463.

(10) Wang, F.; Landau, D. P. Determining the density of states for classical statistical models: A random walk algorithm to produce a flat histogram. Phys. Rev. E 2001, 64, 056101.

(11) Wang, J.-S.; Swendsen, R. H. Transition Matrix Monte Carlo Method. J. Stat. Phys. 2002, 106, 245-285.

(12) Yan, Q.; Faller, R.; de Pablo, J. J. Density-of-states Monte Carlo method for simulation of fluids. J. Chem. Phys. 2002, 116, 8745-8749.

(13) Shell, M. S.; Debenedetti, P. G.; Panagiotopoulos, A. Z. An improved Monte Carlo method for direct calculation of the density of states. J. Chem. Phys. 2003, 119, 94069411.

(14) Errington, J. R. Direct calculation of liquid-vapor phase equilibria from transition matrix Monte Carlo simulation. J. Chem. Phys. 2003, 118, 9915-9925.

(15) Errington, J. R. Evaluating surface tension using grand-canonical transition-matrix Monte Carlo simulation and finite-size scaling. Phys. Rev. E 2003, 67, 012102.

(16) Shen, V. K.; Errington, J. R. Determination of fluid-phase behavior using transitionmatrix Monte Carlo: Binary Lennard-Jones mixtures. J. Chem. Phys. 2005, 122. 
(17) Ganzenmüller, G.; Camp, P. J. Applications of Wang-Landau sampling to determine phase equilibria in complex fluids. J. Chem. Phys. 2007, 127, 154504.

(18) Desgranges, C.; Delhommelle, J. Evaluation of the grand-canonical partition function using expanded Wang-Landau simulations. II. Adsorption of atomic and molecular fluids in a porous material. J. Chem. Phys. 2012, 136, 184108.

(19) Maerzke, K. A.; Gai, L.; Cummings, P. T.; McCabe, C. Simulating Phase Equilibria using Wang-Landau-Transition Matrix Monte Carlo. J. Phys. Conf. Ser. 2014, 487, 012002 .

(20) Mahynski, N. A.; Shen, V. K. Multicomponent adsorption in mesoporous flexible materials with flat-histogram Monte Carlo methods. J. Chem. Phys. 2016, $145,174709$.

(21) Mahynski, N. A.; Blanco, M. A.; Errington, J. R.; Shen, V. K. Predicting lowtemperature free energy landscapes with flat-histogram Monte Carlo methods. J. Chem. Phys. 2017, 146, 074101.

(22) Mahynski, N. A.; Errington, J. R.; Shen, V. K. Temperature extrapolation of multicomponent grand canonical free energy landscapes. J. Chem. Phys. 2017, 14 \%, 054105.

(23) Mahynski, N. A.; Errington, J. R.; Shen, V. K. Multivariable extrapolation of grand canonical free energy landscapes. J. Chem. Phys. 2017, 147, 234111.

(24) Errington, J. R.; Shen, V. K. Direct evaluation of multicomponent phase equilibria using flat-histogram methods. J. Chem. Phys. 2005, 123, 164103.

(25) Rane, K. S.; Murali, S.; Errington, J. R. Monte Carlo Simulation Methods for Computing Liquid-Vapor Saturation Properties of Model Systems. J. Chem. Theory Comput. 2013, 9, 2552-2566.

(26) Wang, F.; Landau, D. P. Efficient, Multiple-Range Random Walk Algorithm to Calculate the Density of States. Phys. Rev. Lett. 2001, 86, 2050-2053. 
(27) Landau, D. P.; Tsai, S.-H.; Exler, M. A new approach to Monte Carlo simulations in statistical physics: Wang-Landau sampling. Am. J. Phys. 2004, 72, 1294-1302.

(28) Smit, B.; Siepmann, J. I. Computer simulations of the energetics and siting of n-alkanes in zeolites. J. Phys. Chem. 1994, 98, 8442-8452.

(29) Vlugt, T. J. H.; Krishna, R.; Smit, B. Molecular Simulations of Adsorption Isotherms for Linear and Branched Alkanes and Their Mixtures in Silicalite. J. Phys. Chem. B 1999, 103, 1102-1118.

(30) Martin, M. G.; Siepmann, J. I. Novel Configurational-Bias Monte Carlo Method for Branched Molecules. Transferable Potentials for Phase Equilibria. 2. United-Atom Description of Branched Alkanes. J. Phys. Chem. B 1999, 103, 4508-4517.

(31) Dubbeldam, D.; Calero, S.; Ellis, D. E.; Snurr, R. Q. RASPA: molecular simulation software for adsorption and diffusion in flexible nanoporous materials. Mol. Simul. 2016, 42, 81-101.

(32) Chopra, R.; Truskett, T. M.; Errington, J. R. On the use of excess entropy scaling to describe single-molecule and collective dynamic properties of hydrocarbon isomer fluids. J. Phys. Chem. B 2010, 114, 16487-16493.

(33) Vlugt, T. J. H.; García-Pérez, E.; Dubbeldam, D.; Ban, S.; Calero, S. Computing the Heat of Adsorption using Molecular Simulations: The Effect of Strong Coulombic Interactions. J. Chem. Theory Comput. 2008, 4, 1107-1118.

(34) Myers, A. L. Chem. Thermodyn. Ind.; 2004; pp 243-252.

(35) Jiang, J.; Furukawa, H.; Zhang, Y.-B.; Yaghi, O. M. High Methane Storage Working Capacity in Metal-Organic Frameworks with Acrylate Links. J. Am. Chem. Soc. 2016, $138,10244-10251$. 
(36) Harris, J. G.; Yung, K. H. Carbon Dioxide's Liquid-Vapor Coexistence Curve And Critical Properties as Predicted by a Simple Molecular Model. J. Phys. Chem. 1995, 99, 12021-12024.

(37) Rappe, A. K.; Casewit, C. J.; Colwell, K. S.; Goddard, W. A.; Skiff, W. M. UFF, a full periodic table force field for molecular mechanics and molecular dynamics simulations. J. Am. Chem. Soc. 1992, 114, 10024-10035.

(38) Shell, M. S.; Debenedetti, P. G.; Panagiotopoulos, A. Z. Flat-histogram dynamics and optimization in density of states simulations of fluids. J. Phys. Chem. B 2004, 108, 19748-19755.

(39) Escobedo, F. A.; Martinez-Veracoechea, F. J. Optimization of expanded ensemble methods. J. Chem. Phys. 2008, 129.

(40) Peng, D.-Y.; Robinson, D. B. A New Two-Constant Equation of State. Ind. Eng. Chem. Fundam. 1976, 15, 59-64.

(41) Paluch, A. S.; Shen, V. K.; Errington, J. R. Comparing the Use of Gibbs Ensemble and Grand-Canonical Transition-Matrix Monte Carlo Methods to Determine Phase Equilibria. Ind. Eng. Chem. Res. 2008, 47, 4533-4541.

(42) Shen, V.; Siderius, D.; Krekelberg, W.; Hatch, H. NIST Standard Reference Simulation Website. http://doi.org/10.18434/T4M88Q.

(43) Pizzi, G.; Cepellotti, A.; Sabatini, R.; Marzari, N.; Kozinsky, B. AiiDA: automated interactive infrastructure and database for computational science. Comput. Mater. Sci. 2016, 111, 218-230.

(44) Lin, L.-C.; Berger, A. H.; Martin, R. L.; Kim, J.; Swisher, J. A.; Jariwala, K.; Rycroft, C. H.; Bhown, A. S.; Deem, M. W.; Haranczyk, M.; Smit, B. In silico screening of carbon-capture materials. Nat. Mater. 2012, 11, 633-641. 
(45) Huck, J. M.; Lin, L.-C.; Berger, A. H.; Shahrak, M. N.; Martin, R. L.; Bhown, A. S.; Haranczyk, M.; Reuter, K.; Smit, B. Evaluating different classes of porous materials for carbon capture. Energy Environ. Sci. 2014, 7, 4132-4146. 


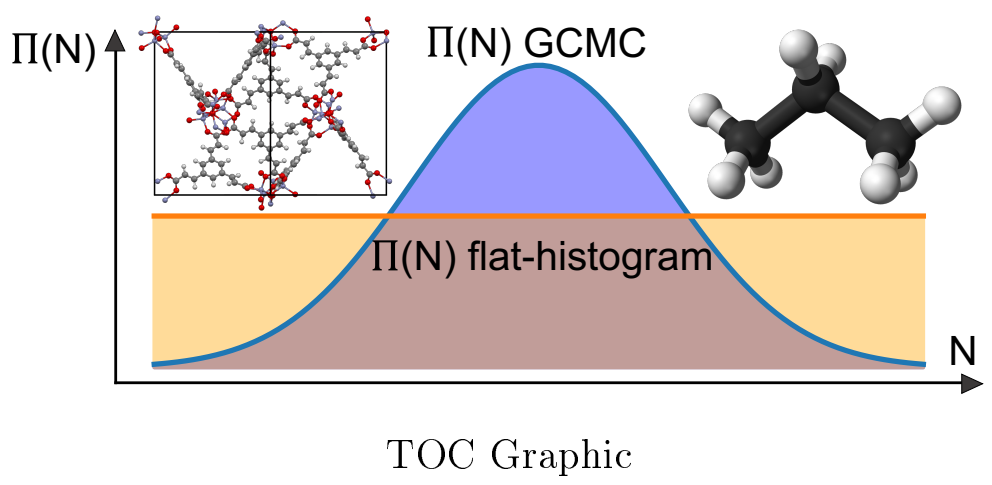

